\title{
A prospective observational cohort study in primary care practices to identify factors associated with treatment failure in Staphylococcus aureus skin and soft tissue infections
}

Grace C. Lee ${ }^{1,2^{*}}$, Ronald G. Hall ${ }^{3,4}$, Natalie K. Boyd ${ }^{1,2}$, Steven D. Dallas ${ }^{5}$, Liem C. Du ${ }^{6}$, Lucina B. Treviño ${ }^{6}$,

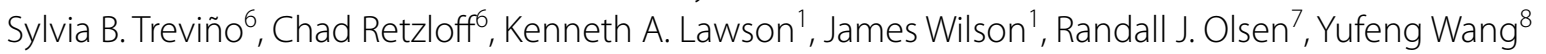
and Christopher R. Frei ${ }^{1,2}$

\begin{abstract}
Background: The incidence of outpatient visits for skin and soft tissue infections (SSTIs) has substantially increased over the last decade. The emergence of community-associated methicillin-resistant Staphylococcus aureus (CA-MRSA) has made the management of S. aureus SSTIs complex and challenging. The objective of this study was to identify risk factors contributing to treatment failures associated with community-associated S. aureus skin and soft tissue infections SSTIS.
\end{abstract}

Methods: This was a prospective, observational study among 14 primary care clinics within the South Texas Ambulatory Research Network. The primary outcome was treatment failure within 90 days of the initial visit. Univariate associations between the explanatory variables and treatment failure were examined. A generalized linear mixed-effect model was developed to identify independent risk factors associated with treatment failure.

Results: Overall, 21\% (22/106) patients with S. aureus SSTIs experienced treatment failure. The occurrence of treatment failure was similar among patients with methicillin-resistant $S$. aureus and those with methicillin-susceptible $S$. aureus SSTIS (19 vs. 24\%; $p=0.70$ ). Independent predictors of treatment failure among cases with S. aureus SSTIs was a duration of infection of $\geq 7$ days prior to initial visit $[a \mathrm{OR}, 6.02(95 \% \mathrm{Cl} 1.74-19.61)]$ and a lesion diameter size $\geq 5 \mathrm{~cm}$ [5.25 (1.58-17.20)].

Conclusions: Predictors for treatment failure included a duration of infection for $\geq 7$ days prior to the initial visit and a wound diameter of $\geq 5 \mathrm{~cm}$. A heightened awareness of these risk factors could help direct targeted interventions in high-risk populations.

Keywords: Staphylococcus aureus, Skin and soft tissue infections, Methicillin-resistant Staphylococcus aureus (MRSA), Epidemiology, Primary care

\footnotetext{
${ }^{*}$ Correspondence: leeg3@uthscsa.edu

2 Pharmacotherapy Education and Research Center, School of Medicine,

The University of Texas Health Science Center, 7703 Floyd Curl Dr, MC

6220, San Antonio, TX 78229-3900, USA

Full list of author information is available at the end of the article
} 


\section{Background}

The incidence of outpatient and emergency department visits for skin and soft tissue infections (SSTIs) has substantially increased with the emergence of community-associated methicillin-resistant Staphylococcus aureus (CA-MRSA) [1]. In the U.S., approximately $80-90 \%$ of SSTIs are due to $S$. aureus [2, 3]. Moreover, treatment failure is common after an initial S. aureus SSTI episode; recurrence rates have exceeded 50\% in some populations [4]. Treatment failure may be multifactorial and can be associated with host factors, disease management, and pathogen features. Two studies set in urgent care and primary care clinics found $35 \%$ of patients with CA-MRSA SSTIs experienced treatment failure and $78 \%$ reported SSTI recurrence $[5,6]$. SSTIs due to CA-MRSA have been implicated to have more serious outcomes compared to community-associated methicillin susceptible $S$. aureus (CA-MSSA) SSTIs; however, there are limited studies evaluating the differences in treatment outcomes in the primary care setting. Furthermore, while there has been a growing body supporting the assessment of early response in treatment failure among hospitalized patients with SSTIs, very little information is available for outpatients [7, 8]. Tools to better identify those who are at higher risk of experiencing treatment failure are needed to better inform treatment decisions in the outpatient setting.

We have recently described the prevalence, treatment characteristics, and costs associated with the management of CA-MRSA SSTIs in South Texas in the primary care setting $[9,10]$. The primary objective of this study was to identify risk factors contributing to S. aureus SSTI treatment failures.

\section{Methods}

\section{Study setting and population}

We performed this investigation among a well-described cohort of patients with SSTIs in the primary care setting. Details of this cohort have been described previously [9-11]. Briefly, this study was conducted in collaboration with fourteen clinics within the South Texas Ambulatory Research Network (STARNet), a practice-based research network (PBRN) composed of 108 urban, suburban, and rural primary care clinics distributed throughout the South Texas region, from 2007 to 2014. Patients were eligible for study enrollment if they provided informed consent, were 18 years of age or older, and presented to one of the participating clinics with an SSTI. Healthcare providers collected a wound sample and patient information (e.g., demographics, infection characteristics, clinical information).

\section{Study design}

We conducted a prospective observational cohort study to determine predictors of treatment failure. Currently, there is no consensus definition of treatment failure. We have based our definition based on prior studies using a proxy of therapeutic endpoints for SSTIs in the outpatient setting [9-14]. Treatment failure was defined as any of the following events within 90 days of their initial visit: (1) need for a new course or change in antibiotic therapy for SSTI, (2) additional incision and drainage, (3) SSTI at a new site, (4) SSTI at the same site, (5) emergency department visit, or (6) hospital admission. First, we compared the rate of treatment failure for patients with MRSA infections to those with MSSA infections. Next, we identified independent risk factors associated with treatment failure by comparing key characteristics in those patients who did and did not experience treatment failure.

\section{Microbiologic analyses}

Samples were plated onto blood agar plates (TSA with $5 \%$ sheep blood; Fisher Scientific, Lenexa, KS) and incubated at $35-37{ }^{\circ} \mathrm{C}$ for $24 \mathrm{~h}$, then sub-cultured to MRSA selective agar (MRSASelect chromogenic agar plates; Bio-Rad Laboratories, Hercules, CA). Latex agglutination tests (StaphAurex ${ }^{\circledR}$; Thermo Fisher Scientific, Lenexa, $\mathrm{KS}$ ), and phenotypic screening tests (cefoxitin) were used for the identification and isolation of MRSA using Vitek 2 AST-GP75 cards (bioMerieux, Durham, NC). Antimicrobial minimum inhibitory concentrations (MICs) were interpreted according to the Clinical and Laboratory Standards Institute document M100-S22 (2012). Multidrug resistance (MDR) was defined as resistant to $>2$ antimicrobial classes. For molecular analysis, multilocus sequence types (MLST) were assigned for 98 isolates using whole genome sequence data according to designated MLST (http://www.mlst.net) as described previously [11].

\section{Data collection and variables}

Clinical information included patient gender, race (Black, White, Other), ethnicity (Hispanic, Non-Hispanic), past medical history (e.g., diabetes, peripheral vascular disease, chronic non-infectious skin disorder, HIV/AIDS, cancer, actively receiving chemotherapy, immunosuppression), health care-related work history, skin infection history, height, infection characteristics (e.g., location, duration, size, deepest tunnel depth, erythema, smell, ulceration, drainage, abscess, satellites), incision and drainage procedures, and antibiotics prescribed. A BMI $\geq 30 \mathrm{~kg} / \mathrm{m}^{2}$ was used to indicate obesity status. A $110 \mathrm{~kg}$ weight cutoff was used to indicate 'high body weight'. 
This is consistent with previous literature associating high body weight with antimicrobial dosing outcomes [15-17]. In addition, this cut-off was internally derived using a Classification and Regression Tree (CART) analysis which found a significant node at $110 \mathrm{~kg}$ that partitioned the data associated with treatment failure.

\section{Statistical analyses}

First, a bivariable analysis was conducted comparing variables between the 'treatment failure' and 'no treatment failure' groups. The Breslow-Day test was used to identify possible effect modification; any $p \leq 0.05$ was considered an effect modifier. A generalized linear mixed-effect model was developed to identify independent risk factors; clinic site was set as the random effect. Covariates included MRSA phenotype, largest diameter size of the wound $\geq 5 \mathrm{~cm}$, and duration of skin infection prior to visit of $\geq 7$ days. Adjusted odds ratios (ORs) and 95\% confidence intervals (CIs) were reported. A $p \leq 0.05$ was used to determine statistical significance. SPSS $22.0^{\circledR}$ (IBM Corp, Armonk, NY) was used for all statistical comparisons.

\section{Results}

Among cases with positive $S$. aureus SSTIs, 106 cases (61\%) had 90-days follow-up information. Overall, 22 (21\%) cases experienced treatment failure. Treatment failure occurred in 19\% (13) of cases with initial MRSA SSTIs and 24\% (9) with MSSA SSTIs $(p=0.70)$. In bivariable analysis, factors associated with treatment failure included Black race, weight $\geq 110 \mathrm{~kg}$, MDR, duration of skin infection prior to visit $\geq 7$ days, lesion diameter $\geq 5 \mathrm{~cm}$, lesion size $\geq 25 \mathrm{~cm}^{2}$, and abscess formation (Table 1). Multivariable analysis showed no significant difference in the likelihood of treatment failure between MRSA and MSSA (aOR, $0.42(0.12-1.42) ; p=0.16$ ). Independent predictors of treatment failure among cases with $S$. aureus SSTIs were duration of skin infection prior to visit $\geq 7$ days [aOR, 6.02 (95\% CI 1.74-19.61)], and a lesion diameter size $\geq 5 \mathrm{~cm}$ [aOR, 5.25 (95\% CI 1.58-17.20)].

Infection location, specific treatment strategies, or prescribed antimicrobial agents were not significantly associated with treatment failure. The proportion of discordant antimicrobial agents prescribed was higher in the treatment failure group than in the no-failure group, but did not reach statistical significance (11 vs. $4 \%, p=0.20$ ) which may be an artifact of the small sample size. MRSA isolates had significantly lower susceptibility to ciprofloxacin $(p<0.01)$ and erythromycin $(p<0.01)$. Two isolates (1 MRSA and 1 MSSA) were D-test positive.

Molecular analysis (Fig. 1) was conducted on 98 isolates: $56 \%(65 / 116)$ of CA-MRSA and $43 \%(32 / 75)$ of
CA-MSSA. All MRSA isolates and 68\% of MSSA isolates were MLST strain type (ST) 8. Other MSSA strain types included: ST5, ST12, ST-15, ST-20, ST-45, and ST-59. Four isolates had undefined MLST designation. Furthermore, $95 \%$ of $S$. aureus SSTI treatment failures were ST- 8 compared to $84 \%$ of cases with no treatment failure $(p=0.32)$.

\section{Discussion}

Over the past 10 years, ambulatory care visits for SSTIs have increased exponentially. The worldwide emergence of CA-MRSA strains has made the management of $S$. aureus SSTIs extremely complicated and challenging [2, $3]$. A clinical approach to the management of $S$. aureus SSTIs is to identify risk factors to predict those who are more likely to experience treatment failure.

Although it has been postulated that CA-MRSA related SSTIs may be associated with worse outcomes, SSTIs caused by MRSA did not have worse outcomes than those caused by MSSA in the South Texas community. Miller et al. found similar 30-day response rates among patients with CA-MRSA and CA-MSSA infection (23 [33\%] of 70 vs. 13 [28\%] of 47 patients; $p=0.55$ ). In addition, patients with CA-MSSA infections were more likely to be re-hospitalized and to subjectively believe that they had not been cured [18]. Moreover, a previous randomized clinical trial of children with suspected $S$. aureus SSTIs found that the incidence of recurrence did not differ between children with MRSA vs. MSSA baseline infections [14]. Our findings further support the notion that the methicillin resistance phenotype is not a reliable predictor for clinical outcomes of community associated $S$. aureus infections. Rather, community associated $S$. aureus should be considered a single entity when evaluating virulence risks.

Identifying predictors for clinical failure can help target more aggressive treatment, monitoring, and decolonization procedures. This study identified that duration of infection 7 days and longer prior to the initial visit was the strongest predictor of treatment failure. This may be related to the natural course of infection that patients were presenting at a time of maximum intensity of inflammation and infection. Moreover, it may be that longer duration of an active S. aureus infection without proper treatment may have increased the likelihood for household or fomite transmission, both which have been shown to be important factors for infection recurrence [19-21]. This finding is in contrast to a recent study that found longer duration of symptoms was among the factors related to early response (at day 3 ). However, the investigation evaluated antibiotic response at day 3 among hospitalized SSTI patients; therefore, may not necessarily be associated with long-term outcomes 
Table 1 Risk factors associated with treatment failure among patients with community-associated S. aureus skin and soft tissue infections

\begin{tabular}{|c|c|c|c|c|c|c|c|}
\hline Characteristic & $\begin{array}{l}\text { Overall, } \\
n=106\end{array}$ & $\begin{array}{l}\text { No failure, } \\
n=84\end{array}$ & $\begin{array}{l}\text { Treatment failure, } \\
\mathrm{n}=22\end{array}$ & OR $(95 \% \mathrm{Cl})$ & $p$ & aOR $(95 \% \mathrm{Cl})$ & $p$ \\
\hline Mean age, years $( \pm S D)$ & $41( \pm 14)$ & $40( \pm 13)$ & $45( \pm 13)$ & & 0.15 & & \\
\hline \multicolumn{8}{|l|}{ Gender } \\
\hline Male & $53(50 \%)$ & $44(52 \%)$ & $9(41 \%)$ & $0.63(0.24-1.63)$ & 0.34 & & \\
\hline \multicolumn{8}{|l|}{ Race/ethnicity } \\
\hline Black & $8(8 \%)$ & $4(5 \%)$ & $4(18 \%)$ & $4.44(1.02-19.47)$ & 0.03 & & \\
\hline Hispanic & $78(74 \%)$ & 64 (76\%) & $14(64 \%)$ & $0.547(0.20-1.49)$ & 0.23 & & \\
\hline Diabetes & $28(26 \%)$ & $21(25 \%)$ & $7(32 \%)$ & $1.40(0.50-3.89)$ & 0.52 & & \\
\hline Obese $(B M I \geq 30)^{b}$ & $50(54 \%)$ & $38(50 \%)$ & $12(71 \%)$ & $2.40(0.77-7.48)$ & 0.12 & & \\
\hline Weight $\geq 110 \mathrm{~kg}$ & $19(20 \%)$ & $12(15 \%)$ & $7(37 \%)$ & $3.21(1.05-9.80)$ & 0.04 & & \\
\hline $\begin{array}{l}\text { Chronic non-infectious skin } \\
\text { disorder }\end{array}$ & $1(1 \%)$ & $1(1 \%)$ & 0 & $0.99(0.97-1.01)$ & 1.00 & & \\
\hline $\begin{array}{l}\text { Immunosuppressed at time } \\
\text { of visit }\end{array}$ & $2(2 \%)$ & $2(2 \%)$ & 0 & $0.98(0.94-1.01)$ & 1.00 & & \\
\hline $\begin{array}{l}\text { Provides healthcare to } \\
\text { others }\end{array}$ & $2(2 \%)$ & $1(1 \%)$ & 0 & $0.99(0.97-1.01)$ & 1.00 & & \\
\hline MRSA phenotype & $68(65 \%)$ & 55 (66\%) & $13(59 \%)$ & $0.87(0.49-1.56)$ & 0.65 & $0.42(0.12-1.42)$ & 0.16 \\
\hline MDR & $29(27 \%)$ & $10(12 \%)$ & $19(86 \%)$ & $2.85(1.07-7.62)$ & 0.03 & & \\
\hline Prior SSTI & 35 (33\%) & $27(32 \%)$ & $8(36 \%)$ & $1.21(0.45-3.20)$ & 0.71 & & \\
\hline Prior antibiotic history & $16(15 \%)$ & $11(13 \%)$ & $5(23 \%)$ & $1.95(0.60-6.36)$ & 0.32 & & \\
\hline $\begin{array}{l}\text { Duration of infection prior } \\
\text { to visit } \geq 7 \text { days }\end{array}$ & $48(48 \%)$ & $32(40 \%)$ & $16(76 \%)$ & $4.80(1.59-14.41)$ & $<0.01$ & $6.02(1.74-20.87)$ & $<0.01$ \\
\hline \multicolumn{8}{|l|}{ Severity } \\
\hline Largest diameter $\geq 5 \mathrm{~cm}$ & $49(48 \%)$ & $34(42 \%)$ & $15(71 \%)$ & $3.53(1.24-10.02)$ & 0.01 & $5.25(1.58-17.42)$ & $<0.01$ \\
\hline Lesion area $\geq 25 \mathrm{~cm}^{2}$ & $37(35 \%)$ & $24(29 \%)$ & $13(59 \%)$ & $3.55(1.34-9.39)$ & 0.01 & & \\
\hline \multicolumn{8}{|l|}{ Infection characteristics } \\
\hline Erythema & $78(74 \%)$ & $61(74 \%)$ & $17(77 \%)$ & $1.23(0.40-3.72)$ & 0.72 & & \\
\hline Drainage & $56(53 \%)$ & $45(54 \%)$ & $11(50 \%)$ & $0.84(0.33-2.16)$ & 0.72 & & \\
\hline Ulceration & $30(29 \%)$ & $22(27 \%)$ & $8(36 \%)$ & $1.58(0.59-4.29)$ & 0.43 & & \\
\hline Abscess & $76(72 \%)$ & $56(67 \%)$ & $20(91 \%)$ & $4.82(1.05-22.14)$ & 0.03 & & \\
\hline \multicolumn{8}{|l|}{ Location } \\
\hline Lower extremity & $35(33 \%)$ & $26(31 \%)$ & $9(41 \%)$ & $1.54(0.59-4.06)$ & 0.38 & & \\
\hline Head/neck/face & $11(10 \%)$ & $10(12 \%)$ & $1(5 \%)$ & $0.35(0.40-2.91)$ & $0.45^{*}$ & & \\
\hline Trunk & $24(23 \%)$ & $17(20 \%)$ & $7(32 \%)$ & $1.84(0.65-5.22)$ & $0.26^{*}$ & & \\
\hline Axilla & $13(12 \%)$ & $11(13 \%)$ & $2(9 \%)$ & $0.66(0.14-3.24)$ & $0.61^{*}$ & & \\
\hline Upper extremity & $6(6 \%)$ & $5(6 \%)$ & $1(5 \%)$ & $0.75(0.08-6.79)$ & $1.00^{*}$ & & \\
\hline Groin/buttock & $17(16 \%)$ & $14(17 \%)$ & $3(14 \%)$ & $0.79(0.21-3.03)$ & $1.00^{*}$ & & \\
\hline \multicolumn{8}{|l|}{ Treatment } \\
\hline I\&D only & $4(4 \%)$ & $3(4 \%)$ & $1(5 \%)$ & $1.01(0.91-1.12)$ & 1.00 & & \\
\hline I\&D + antibiotics & 57 (59\%) & $43(51 \%)$ & $14(64 \%)$ & $1.30(0.68-2.51)$ & 0.41 & & \\
\hline Antibiotics only & $32(33 \%)$ & $27(36 \%)$ & $5(24 \%)$ & $0.84(0.63-1.13)$ & 0.29 & & \\
\hline \multicolumn{8}{|l|}{ Antibiotics } \\
\hline $\begin{array}{l}\text { Trimethoprim-sulfameth- } \\
\text { oxazole }\end{array}$ & 81 (76\%) & $65(77 \%)$ & $16(73 \%)$ & $0.78(0.27-2.27)$ & 0.65 & & \\
\hline
\end{tabular}

including post-treatment failure and recurrence that were evaluated in the current study [7]. Importantly, this finding further supports the proposition that time to effective treatment is essential in the management of SSTIs, and possibly, that patients presenting with longer duration ( $\geq 7$ days) of infections may require more aggressive measures and/or follow-up monitoring.

In addition, a lesion diameter of greater than $5 \mathrm{~cm}$ was associated with treatment failure. Lesion size has been used as a proxy for severity in several syndromes of 
Table 1 continued

\begin{tabular}{|c|c|c|c|c|c|c|c|}
\hline Characteristic & $\begin{array}{l}\text { Overall, } \\
n=106\end{array}$ & $\begin{array}{l}\text { No failure, } \\
n=84\end{array}$ & $\begin{array}{l}\text { Treatment failure, } \\
n=22\end{array}$ & OR $(95 \% \mathrm{Cl})$ & $p$ & aOR $(95 \% \mathrm{CI})$ & $p$ \\
\hline Doxycycline & $12(11 \%)$ & $9(11 \%)$ & $3(14 \%)$ & $1.32(0.32-5.34)$ & 0.71 & & \\
\hline Clindamycin & $7(7 \%)$ & $5(6 \%)$ & $2(9 \%)$ & $1.58(0.26-8.75)$ & 0.63 & & \\
\hline Cephalexin & $9(9 \%)$ & $7(8 \%)$ & $2(9 \%)$ & $1.10(0.21-5.71)$ & 1.00 & & \\
\hline Discordant therapy & $5(5 \%)$ & $3(4 \%)$ & $2(11 \%)$ & $2.82(0.44-18.24)$ & 0.26 & & \\
\hline
\end{tabular}

Note there were no cases of patients with the peripheral vascular disease, human immunodeficiency virus, cancer, and receipt of chemotherapy

MRSA methicillin resistant S. aureus, MSSA methicillin susceptible $S$. aureus, SD standard deviation, OR odds ratio, $\mathrm{Cl}$ confidence interval, aOR adjusted odds ratio, SSTI skin and soft tissue infection, $B M I$ body mass index, I\&D incision and drainage

* Fishers exact test was used

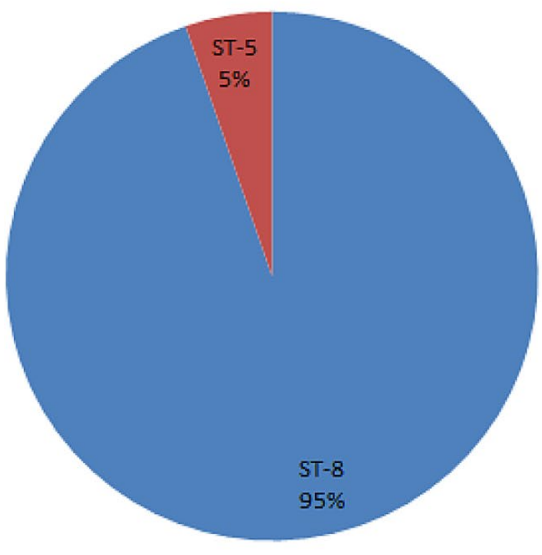

Failure

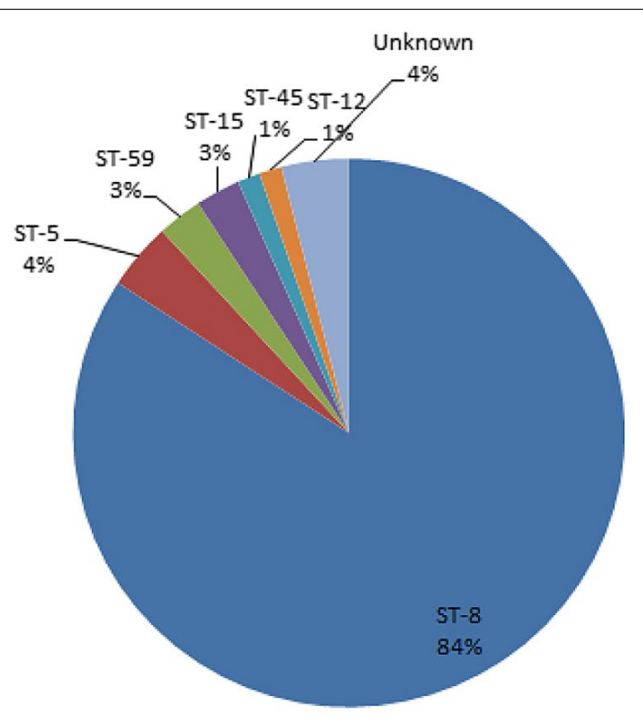

No Failure

Fig. 1 Multilocus sequence typing of Staphylococcus aureus isolates associated with treatment failure or no treatment failure

skin infections, including necrotizing fasciitis and surgical site infections [22]. The $5 \mathrm{~cm}$ threshold used in this study was based on data from an observational study, in which abscesses larger than $5 \mathrm{~cm}$ were associated with treatment failure [23]. This investigation found a significant predictor of hospitalization on the first follow-up was having an infected area $>5 \mathrm{~cm}$ in diameter at the initial evaluation (33\% of patients with diameter $>5 \mathrm{~cm}$ were later hospitalized vs. none with diameter $\leq 5 \mathrm{~cm}$; $p=0.004)$. Other studies have also identified lesion size as an important indicator for severity and suggest treatment stratification approaches [24-26]. In the most recent guidelines for acute bacterial skin and skin structure infection (ABSSSI) clinical trials, the FDA recommends an evaluation of lesion size as a more quantitative assessment of infection severity and the change in lesion size at 48 to $72 \mathrm{~h}$ as the new primary endpoint [27].
Our findings provide further support to assess initial lesion sizes to predict infection severity and treatment outcomes.

Specific treatment strategies or type of prescribed antimicrobial agents were not found to be independent factors associated with treatment failure. It should be noted that because of the limited sample size, this study was not designed to perform direct comparisons of treatment approaches and antibiotic therapies. Larger investigations are needed to compare the role of treatment strategies and antibiotic regimens.

In our molecular analyses, ST-8 was the predominant strain type and was more likely to be found in patients whose treatment failed. However, this $11 \%$ absolute difference did not reach statistical significance due to the size of our cohort. This might suggest that the pathogenicity of community associated S. aureus may be based 
on the genetic background or lineage rather than the methicillin susceptibility phenotype. A more thorough investigation on the genomic characteristics of these strains is currently underway.

There are limitations to this study. First, we did not account for social and behavioral risk factors that may be associated with $S$. aureus SSTIs and/or clinical outcomes. Second, we used laboratory diagnosis to identify S. aureus cases. Patients presenting with SSTIs with no culture or were culture-negative may have different characteristics. The small sample size limited the ability to identify risks associated with lower exposures. Compliance of antimicrobials prescribed was not assessed. Finally, there may be limited generalizability to other regions outside of South Texas. To our knowledge, this is the first prospective study evaluating the clinical and epidemiological factors of $S$. aureus SSTI treatment failure in the primary care setting, adding important findings to the sparse literature in this growing population.

\section{Conclusions}

The rates of treatment failure were similar among patients with CA-MRSA SSTIs to those with CA-MSSA SSTIs. Independent predictors for treatment failure included a duration of infection for greater than 7 days prior to the initial visit and a wound diameter of $\geq 5 \mathrm{~cm}$. A heightened awareness of these risk factors could help direct clinical management and public health interventions in high-risk populations. Future large prospective investigations are required to validate these findings and to assess optimal treatment approaches.

\footnotetext{
Abbreviations

SSTIs: skin and soft tissue infections; CA-MRSA: community-associated methicillin resistant S. aureus; CA-MSSA: community-associated methicillin susceptible S. aureus; STARNet: South Texas Ambulatory Research Network; PBRN: practice-based research network; MICs: minimum inhibitory concentrations; MDR: multidrug resistance; MLST: multilocus sequence type; CART: classification and regression tree; ORs: odds ratios; Cls: confidence intervals; aOR: adjusted odd ratio; SD: standard deviation; BMI: body mass index; I\&D: incision and drainage; ST: strain type.
}

\section{Authors' contributions}

Conception and design of work: GCL and CRF; Data collection: GCL, SDD, LCD, LBT, SBT, CRF; Data analysis and interpretation: GCL, RGH, CRF; Drafting of manuscript: GCL; Critical revision of manuscript: RGH, NKB, KAL, JAW, RJO, YW, CRF. All authors read and approved the final manuscript.

\footnotetext{
Author details

${ }^{1}$ College of Pharmacy, University of Texas at Austin, Austin, TX, USA. ${ }^{2}$ Pharmacotherapy Education and Research Center, School of Medicine, The University of Texas Health Science Center, 7703 Floyd Curl Dr, MC 6220, San Antonio, TX 78229-3900, USA. ${ }^{3}$ School of Pharmacy, Texas Tech University Health Sciences Center, Dallas, TX, USA. ${ }^{4}$ Dose Optimization and Outcomes Research (DOOR) Program, Dallas, TX, USA. ${ }^{5}$ Department of Clinical Laboratory Sciences, School of Health Professions, University of Texas Health Science Center, San Antonio, TX, USA. ${ }^{6}$ South Texas Ambulatory Research Network, The University of Texas Health Science Center, San Antonio, TX, USA. ${ }^{7}$ Department of Pathology and Genomic Medicine, Houston Methodist Hospital and Research Institute,
}

Houston, TX, USA. ${ }^{8}$ Department of Biology, The University of Texas San Antonio, San Antonio, TX, USA.

\section{Acknowledgements}

The authors would like to thank their South Texas Ambulatory Research Network (STARNet) and Area Health Education Center (AHEC) colleagues, including Paula Winkler, who assisted with the administrative aspects of the study. We also thank Khine Tun for editing and formatting the manuscript.

\section{Competing interests}

The authors declare that they have no competing interests.

\section{Declaration}

The University of Texas Health Science Center Institutional Review Board granted approval for this study.

\section{Funding statement}

This study was funded by an investigator-initiated research grant from Pfizer to Dr. Frei. Dr. Frei was also supported by the U.S. National Institutes of Health in the form of a KL2 Career Development Award (NIH/NCRR 5KL2 RR025766, PI-Robert Clark).

Received: 31 August 2016 Accepted: 18 November 2016

Published online: 22 November 2016

\section{References}

1. Pallin DJ, Egan DJ, Pelletier AJ, Espinola JA, Hooper DC, Camargo CA Jr. Increased US emergency department visits for skin and soft tissue infections, and changes in antibiotic choices, during the emergence of community-associated methicillin-resistant Staphylococcus aureus. Ann Emerg Med. 2008:51:291-8.

2. Ray GT, Suaya JA, Baxter R. Incidence, microbiology, and patient characteristics of skin and soft-tissue infections in a U.S. population: a retrospective population-based study. BMC Infect Dis. 2013;13:252.

3. Ray GT, Suaya JA, Baxter R. Microbiology of skin and soft tissue infections in the age of community-acquired methicillin-resistant Staphylococcus aureus. Diagn Microbiol Infect Dis. 2013;76:24-30.

4. Creech CB, Al-Zubeidi DN, Fritz SA. Prevention of recurrent Staphylococcal skin infections. Infect Dis Clin North Am. 2015;29:429-64.

5. Frei $C R$, Miller ML, Lewis JS 2 nd, et al. Trimethoprim-sulfamethoxazole or clindamycin for community-associated MRSA (CA-MRSA) skin infections. J Am Board Fam Med. 2010:23:714-9.

6. Parchman ML, Munoz A. Risk factors for methicillin-resistant Staphylococcal aureus skin and soft tissue infections presenting in primary care: a South Texas Ambulatory Research Network (STARNet) study. J Am Board Fam Med. 2009:22:375-9.

7. Bruun T, Oppegaard O, Hufthammer KO, et al. Early response in celIulitis: a prospective study of dynamics and predictors. Clin Infect Dis. 2016;15:1034-41.

8. Amara S, Adamson RT, Lew I, et al. Clinical response at Day 3 of therapy and economic outcomes in hospitalized patients with acute bacterial skin and skin structure infection (ABSSSI). Curr Med Res Opin. 2013:29:869-77.

9. Forcade NA, Parchman ML, Jorgensen JH, et al. Prevalence, severity, and treatment of community-acquired methicillin-resistant Staphylococcus aureus (CA-MRSA) skin and soft tissue infections in ten medical clinics in Texas: a South Texas Ambulatory Research Network (STARNet) study. J Am Board Fam Med. 2011;24:543-50.

10. Labreche MJ, Lee GC, Attridge RT, et al. Treatment failure and costs in patients with methicillin-resistant Staphylococcus aureus (MRSA) skin and soft tissue infections: a South Texas Ambulatory Research Network (STARNet) study. J Am Board Fam Med. 2013;26:508-17.

11. Lee GC, Long SW, Musser JM, et al. Comparative whole genome sequencing of community-associated methicillin-resistant Staphylococcus aureus sequence type 8 from primary care clinics in a Texas community. Pharmacotherapy. 2015;35:220-8. 
12. Bocchini CE, Mason EO, Hulten KG, et al. Recurrent community-associated Staphylococcus aureus infections in children presenting to Texas Children's Hospital in Houston Texas. Pediatr Infect Dis J. 2013;32:1189-93.

13. Fritz SA, Hogan PG, Hayek G, et al. Household versus individual approaches to eradication of community-associated Staphylococcus aureus in children: a randomized trial. Clin Infect Dis. 2012;54:743-51.

14. Kaplan SL, Forbes A, Hammerman WA, et al. Randomized trial of "bleach baths" plus routine hygienic measures vs. routine hygienic measures alone for prevention of recurrent infections. Clin Infect Dis. 2014;58:679-82.

15. Rubino CM, Van Wart SA, Bhavnani SM, Ambrose PG, McCollam JS, Forrest A. Oritavancin population pharmacokinetics in healthy subjects and patients with complicated skin and skin structure infections or bacteremia. Antimicrob Agents Chemother. 2009;53:4422-8.

16. Falagas ME, Karageorgopoulos DE. Adjustment of dosing of antimicrobial agents for bodyweight in adults. Lancet. 2010;375:248-51.

17. Gillon JE, Cassat JE, Di Pentima MC. Validation of two vancomycin nomograms in patients 10 years of age and older. J Clin Pharmacol. 2014;54:35-8.

18. Miller LG, Quan C, Shay A, et al. A prospective investigation of outcomes after hospital discharge for endemic, community-acquired methicillinresistant and -susceptible Staphylococcus aureus skin infection. Clin Infect Dis. 2007;44:483-92.

19. Miller LG, Diep BA. Clinical practice: colonization, fomites, and virulence: rethinking the pathogenesis of community-associated methicillin-resistant Staphylococcus aureus infection. Clin Infect Dis. 2008;46:752-60.
20. Uhlemann AC, Dordel J, Knox JR, et al. Molecular tracing of the emergence, diversification, and transmission of $S$. aureus sequence type 8 in a New York community. Proc Natl Acad Sci USA. 2014;111:6738-43.

21. Uhlemann AC, Kennedy AD, Martens C, Porcella SF, Deleo FR, Lowy FD. Toward an understanding of the evolution of Staphylococcus aureus strain USA300 during colonization in community households. Genome Biol Evol. 2012:4:1275-85.

22. Stevens DL, Bisno AL, Chambers HF, et al. Practice guidelines for the diagnosis and management of skin and soft tissue infections: 2014 update by the infectious diseases society of America. Clin Infect Dis. 2014;59:147-59.

23. Lee MC, Rios AM, Aten MF, Mejias A, Cavuoti D, McCracken GH Jr, Hardy RD. Management and outcome of children with skin and soft tissue abscesses caused by community-acquired methicillin-resistant Staphylococcus aureus. Pediatr Infect Dis J. 2004;23:123-7.

24. Miller LG, Daum RS, Chambers HF. Antibacterial treatment for uncomplicated skin infections. N Engl J Med. 2015;372:2460.

25. Miller LG, Daum RS, Creech CB, et al. Clindamycin versus trimethoprimsulfamethoxazole for uncomplicated skin infections. N Engl J Med. 2015;372:1093-103.

26. Eron LJ, Lipsky BA, Low DE, et al. Managing skin and soft tissue infections: expert panel recommendation. J Antimicrob Chemother. 2003;52(Suppl 1):i3-17.

27. Itani KM, Shorr AF. FDA guidance for ABSSSI trials: implications for conducting and interpreting clinical trials. Clin Infect Dis. 2014;58(1):S4-9.

\section{Submit your next manuscript to BioMed Central and we will help you at every step:}

- We accept pre-submission inquiries

- Our selector tool helps you to find the most relevant journal

- We provide round the clock customer support

- Convenient online submission

- Thorough peer review

- Inclusion in PubMed and all major indexing services

- Maximum visibility for your research

Submit your manuscript at www.biomedcentral com/submit 